\title{
Assessment of Object Segmentation Techniques for Object Based Image Retrieval
}

\author{
LaxmideviNoolvi, Hema N, M V Sudhamani
}

\begin{abstract}
Objects relates more to human perception than any other attributes of an image. Image segmentation is a significant image processing technique to get the objects from complex image background. This work assesses the techniques of segmentation from basic global thresholding, edge based methods up to the advanced techniques such as K-means, Active Contour Model (Snakes) segmentation approaches. Later, results are post processed with the help of morphological operations and make them suitable for object based image retrieval. It also provides the comparative analysis and empirical assessment of performance of the proposed modified segmentation approaches.
\end{abstract}

Keywords- OBIR, image segmentation, active contours, K-means image segmentation.

\section{INTRODUCTION}

The general method of image segmentation is to partition an image into regions which are having similar color, texture or having similar features. The image segmentation is very first step, which delete the surplus attributes from the image for the further processing simplification. The segmentation is one of the most difficult yet very significant in the field of image processing. There is no one standard segmentation technique present for all the type of image categories. Choosing a suitable image segmentation technique is highly dependent on the problem statement and the category of the image.

The object based image retrieval systems, requires object segmentation as a preliminary step. Suppose if we look into any image, immediately human beings observe the objects because perceptually they relates more than anything present in an image. For example, either it may be persons, animals or birds etc. The segmentation of the object is very complex because the semantic objects does not always corresponds homogeneous regions of images.

This paper explains the four techniques of image segmentation suitable for object based image retrieval systems. It includes two segmentation techniques such as global threshold based and edge based segmentation methods for simple centred object images.

Revised Manuscript Received on December 15, 2019.

LaxmideviNoolvi*, CSE, RNSIT, Bangalore, India. Email: laxmidevinoovli@gmail.com

Hema N, ISE, RNSIT, Bangalore, India. Email: hemashekar05@gmail.com

Dr. M V sudhamani, ISE, RNSIT, Bangalore, India. Email: mvsudha_raj@hotmail.com

Further the advanced segmentation methods like active contours or snake's method and K-means image segmentation are elaborated here. These four algorithms are then compared and analysed for best approach for object based image retrieval systems.

The paper is organized as follows. In the section 2 the Literature review of different segmentation methods are explained. Techniques of object segmentation from the image background are given in section 3. Experiments and Results are given in section 4 . In section 5 comparative study of segmentation algorithms explained with their precincts and utility, it also provides performance evaluation of segmentation algorithms with empirical measures. Finally paper ends up with concluding the work.

\section{LITERATURE REVIEW}

Image segmentation is a hotspot research area in computer vision today; basically it is dividing the image into non-intersecting regions based on some criteria. The region corresponds to similar pixel values, textures or homogeneous pixels with common features. Enormous literature we can find in the area of image segmentation. In [1, 2], the fundamental approaches of the image segmentation methods such as based on regions and based on edges present in the image are explained. It also explains the segmentation using clustering and weakly-supervised Learning methods using convolution neural network.

In [3], the simple and effective segmentation of images using k-means clustering method which is applied for making clusters of similar intensity values. First step is to convert image in to Lab color space and make the image independent from light effect by setting the luminance value to particular values. After that adaptive k-means algorithm is applied and morphological operations have been performed for segmenting maximally connected region present in an image.

In [4], used the Gradient Vector Field (GVF) active contour for the segmentation and introduced the new automatic initializing the initial boundary by using centric of corners. In [5], method is based on the assuming objects of interest situated at image center and has similar intensity distribution. First it locates the significant area of color distribution using Default Attention Window (DAW) and region merging algorithm has been applied to segment object.

In [6], the novel method of object segmentation in two steps, first step filters the image with three circular adaptive filters which are based on the texture patterns. In second step regions with similar texture which are adjacent are merged and regions are assigned with different labels. 
Finally small noisy regions are deleted by using morphological operations. In [7],new segmentation using Quaternion Transform technique for salient region detection is proposed.In [8], proposed the interesting hybrid technique of object segmentation from image using JSEG segmentation followed by active contour models. Here the JSEG segmentation provides segmentation of overall image with grouping the dominant color quantization. Then the main object is detected with the help of active contour snake's model.

\section{TECHNIQUES OF OBJECT SEGMENTATION}

In this paper, we present four algorithms for object segmentation which are suitable for object based image retrieval systems are discussed here. First two methods are very simple yet very useful for the object based segmentation with simple background information. Those fundamental methods are listed below,

\section{A. Global Threshold based object segmentation with region area filtering}

B. Edge based object segmentation with convex hull.

Another two improved approaches of object segmentation are,

C. k-means image segmenting with morphological
operations

D. Active Contour Snake's model for object segmentation.

Following sub sections explain details of the above mentioned algorithms.

\section{A. Global Threshold based object segmentation with region area filtering}

Thresholding is very normally used method of segmentation in many applications of image processing. It is computationally quite easy and simple method it can be applicable for images with uniform background. In threshold method of segmentation, discrimination between the intensity distributions is pined as one or more values [1]. These values are used for separating the image pixel values into different set of intensity groups that corresponds to non overlapping regions.

Threshold based segmentation can be global and local in the former method which uses the single value for isolating objects from background and in the later method multiple threshold values are used to group the pixel values into different regions [2]. In the proposed segmentation algorithm we use global thresholding method. The general global thresholding equation is given in (1).

$$
B(x, y)=\left\{\begin{array}{ll}
G(x, y) \geq T & 1 \\
G(x, y)<T & 0
\end{array}(1)\right.
$$

$\mathrm{G}$ is original gray image, $\mathrm{x}$ and $\mathrm{y}$ are coordinates, $\mathrm{T}$ is threshold value, B is binary image after applying threshold, where 1 represents object pixels and 0 represents background pixels.
The main principle that lies in global thresholding is calculation automatic threshold based on the maximum interclass distances, which is called as Otsu's method [9]. After thresholding we get black and white image where white represents the object region and black corresponds to background. But using thresholding does not cover the entire object present in the image because it depends on the intensity values only, spatial distribution of pixel values ignored. The resultant image may contain holes inside object region. In order to get whole object region we have to post process the results to integrate the objects regions to one unit by simple image filling operations where holes in the threshold image are filled with white pixel values. At last in order to localize the object we find the region with maximum area as object of interest because objects have larger number of pixels as compared to small redundant regions in an image.

Algorithm for finding object of interest using thresholding is summarized as follows;

Algorithm 1: Finding object of interest using thresholding and region area filtering.

Input: Color Image.

Output: Image with object of interest marked.

1. Convert input image to gray image.

2. Obtain the threshold image using Otsu's thresholding method.

$$
\eta(T)=\frac{\sigma_{B}^{2}(T)}{\sigma_{G}^{2}}(2)
$$

In the equation (2) $\eta(T)$ is measure of separation with threshold value $\mathrm{T}, \sigma_{G}^{2}$ global intensity variance and $\sigma_{B}^{2}$ isbetween-class intensity variance.

3. Region filling operation to get connected object.

4. Calculate area of each connected regions in image and locate the region with maximum area which corresponds to object in an image.

\section{B. Edge based object segmentation with convex hull}

Edges are very important properties of images. In [10, 11], explains all the methods in detail with algorithms for edge extraction from an image. Edge is hasty change in intensity values in image. Basically there are three different kind of edges; ramp edge, step edge, and roof edge. The edge pixels can be detected with help of local gradient operators such as Robert's cross operator, Sobel, Prewitt operators and Laplacian of Gaussianetc. The image is convoluted with gradient operators then by localizing maximum and minimum values of first or second order derivative true edges are extracted. Canny edge detector is best standard edge detector it is capable of detecting both strong and weak edges.

The second algorithm we add novelty to object segmentation by detecting the edges using canny edge detector followed by convex hull formation for edge pixels. 
This convex hull it forms the compaction around the object and gives the polygon representation of objects in images. The detailed different convex hull formation algorithms are explained in [12]. This technique is very simple and segment objects from images properly.

Algorithm for finding object of interest using Edge detection is summarized as follows;

Algorithm 2: Edge based object segmentation with convex hull.

Input: Color Image.

Output: Image with object of interest marked.

1. Convert input image to gray image.

2. Detect the edges present in image using canny edge detection method.

3. Form the convex hull by the edge pixels detected by edge detector.

4. Calculate area of each connected regions in image and locate the region with maximum area which corresponds to object in an image.

\section{K-means Image segmenting with Morphological operations}

Through advancement of new technologies and new way of visualizing the pixel information, many methods of image segmentation are emerged. Hence, with the knowledge spread in many literature proposed the clustering methods that can be applied for grouping the intensity distribution present in an image [3]. K-means image segmentation is one of the best algorithms, many have refined its sequence of processing for their particular desire, and classical method of clustering method of $\mathrm{k}$-means is dealt in [1].

In this method object segmentation is done using the k-means image segmentation and then post processing the results with morphological operations to get the whole object regions of image is carried out. First step is to group or cluster the similar pixel intensity into some set of regions by the help of k-means algorithm using appropriate initial values for cluster centers, and then by using basic image thresholding technique obtained the binary image. In the resultant image object pixels do not corresponds to homogeneous pixel values, it is because the $\mathrm{k}$-means clustering method does not considers spatial information of pixels. It only does clusters based on intensity values of pixels. In other words similar pixel values does not always situated in one place in image they may be scattered but they are having same properties. Finally to get whole object pixels morphological region filling is applied on threshold image. Then maximum connected region is identified and localized as object of interest.

Algorithm for finding object/region of interest using $\mathrm{K}$-means is summarized as follows;

Algorithm 3: Finding object/region of interest using k-means and morphological operations.

Input: Color Image, Number of clusters k.

Output: Image with object of interest marked.

1. Initialize cluster centers to $\mathrm{k}$.

2. The distance D is calculated, between the cluster center and the each pixel value in an image, using the

equation (3)

$$
D=\left|P(x, y)-C_{k}\right|
$$

Where $P(x, y)$ intensity value at location $x$ and $y . C_{k}$ is cluster center value.

3. Based on distance D Assign all the pixels to the nearest cluster centre.

4. New position of the cluster centers are calculated using the equation (4).

5. $\mathrm{C}_{\mathrm{k}}=\frac{1}{\mathrm{k}} \sum_{\mathrm{y} \in \mathrm{C}_{\mathrm{k}}} \sum_{\mathrm{x} \in \mathrm{C}_{\mathrm{k}}} \mathrm{P}(\mathrm{x}, \mathrm{y})$

6. Process repeats until no changes in the center centers values. Remap the cluster pixels into image.

7. Threshold the image using appropriate threshold value.

8. Use morphological filling operation to fill the holes and form the whole object region.

\section{Active Contour Snake's model for object segmentation}

Active contours are the new approach for the image segmentation. The basic principle embedded in active contours is deformable contour that is explained in [13].The active contour snakes and level set method are two major categories of active contour. Former i.e. snakes method is based on energy minimization function of primarily initialized contour and then iteratively locate the final boundary of object. One of the challenging tasks in active contour snakes is knowledge about the initial boundary around the object. The initial boundary can be drawn around object manually and this work is a tedious job.

The mathematical representations of energy minimization function for active contour snakes is given in general form as follows,

Contour is set of points,

$$
\mathrm{C}=\left(\mathrm{x}_{0}, \mathrm{y}_{0}, \mathrm{x}_{1}, \mathrm{y}_{1}, \ldots, \mathrm{x}_{\mathrm{n}-1}, \mathrm{y}_{\mathrm{n}-1}\right)
$$

Energy function of curve or snake is given in equation (6).

$$
\mathrm{E}=\mathrm{E}_{\mathrm{i}}+\mathrm{E}_{\mathrm{e}}
$$

Where $\mathrm{E}$ is total energy of contour and $\mathrm{E}_{\mathrm{i}}$ is internal energy of contour represents elasticity. External energy $E_{e}$ of contour represents shape of the contour or stiffness. The detail and simplified formula for total energy function is given in equation (7).

$$
E(C)=\alpha \sum_{i=0}^{n-1}\left(x_{i+1}-x_{i}\right)^{2}+\left(y_{i+1}-y_{i}\right)^{2}
$$




$$
-\sum_{i=0}^{n-1}\left|I_{x}\left(x_{i}, y_{i}\right)\right|^{2}+\left|I_{y}\left(x_{i}, y_{i}\right)\right|^{2}
$$

In the equation (7), $\alpha$ is relative weight factor and first term of summation corresponds to internal energy and second term is external energy of contour C.The $\mathrm{I}_{\mathrm{x}}$ and $\mathrm{I}_{\mathrm{y}}$ are gradient image in the $\mathrm{x}$ and $\mathrm{y}$ directions respectively hence they corresponds to edge information of image. The objective of snakes is to find the minimum value of $\mathrm{E}$ using gradient descent in order to iteratively locate final boundary of object present in an image.

In this paper, the above discussed algorithm which segment the object of interest by using active contour snake's model with automatic initialization of initial boundary of objects. Automatic initialization of initial contour is explained in [4]. In this work the automatic initialization of initial boundary by drawing the bounding box around the object is done. Then we have applied the active contour snake method which will iteratively converge to the exact true boundary of object with the help of energy minimization function which is explained in equation (7).

Algorithm for finding object of interest using active contours snakes with automatic initialized object boundary is as follows;

Algorithm 4: Finding object of interest using active contours snakes.

Input: Color Image.

Output: Image with object of interest marked.

1. Initialize the snake boundary points which is bounding box generated around the object detected (resultant image of Algorithm 2).

2. The locations of next snake points are calculated by the local minimum energy term $E$ with the help of equation (7).

3. After we iteratively reach final minimum energy value the contour stops converging when points on the contour lined up on edge pixels.

4. Resultant image is the segmented object of interest.

\section{EXPERIMENTAL RESULTS}

In this work we have considered two set of object based images for our experimentation, in that first set of images are taken from COIL-100 database of objects and another set of images are general object based images collected from web. The Fig. 1 shows the results of four proposed algorithms for segmentation of objects from first set of images. Fig. 2 shows the results of four proposed algorithms for segmentation of objects for six different categories of objects with simple varying background taken from second set of images. The threshold based method of object segmentation is easy and facilitates the object retrieval with constant gray background such as black or white. The edge and convex hull based method gives much pretty results for images with some little varying background. The K-means gives acceptable results with combining techniques of clustering the intensities, thresholding and then region filling. The active contour model of snakes gives the object segmentation with appropriate shape representation for objects. The K-means method demonstrates best result of segmentation with highest similarity measure.

\section{COMPARATIVE STUDY OF ALGORITHMS}

There has been plenty of work presented on many techniques of image segmentation over the last three decades. Many techniques have their own advantages and limitations. Based on the application of their problem domain many researchers have presented the comparative study $[14,15]$. The above section gives the detailed algorithms for object segmentation from an image. All these algorithms are constructive for object retrieval systems which are prominently based on the objects present in images. There are two types of comparative study; analysis based comparison and empirical method of comparison.

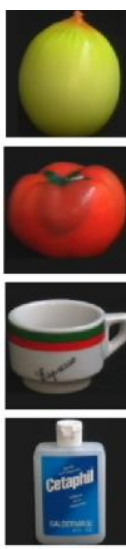

(a) (b)
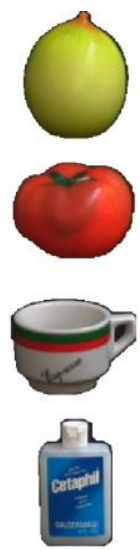

(c)
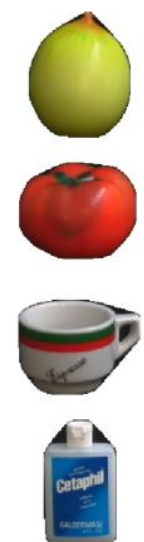

(d)
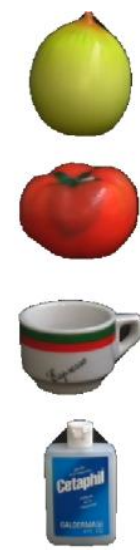

(e)

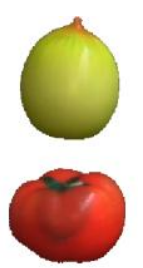

Fig 1: Results of four algorithms (a) Input image (b)

Threshold segmentation (c) Edge segmentation (d) K-means segmentation (e) Active Contour's segmentation.

In the empirical method of comparison it is based on statistical measures calculated over the segmented image and ground truth image. In this paper, we use Jaccard Similarity Coefficient (JSC) and Dice Similarity Coefficient (DSC) [16, 17] to evaluate the performance of the proposed segmentation algorithms. The Table-I shows the experimental values of JSC and DSC for all the four algorithms with COIL-100 sample images. It is observed that the K-means method of segmentation gives the better values as $\mathrm{JSC}=95 \%$ and DSC $=95 \%$. The Active contour Snake method gives the second best results i.e. JSC $=94 \%$ and DSC $=93 \%$. The threshold method showed the values as JSC = $93 \%$ and DSC $=94 \%$. While the edge and convex hull combined method gives comparatively low values such as JSC $=92 \%$ and DSC $=91 \%$. Similarly, The Table-II provides the experimental values of JSC and DSC for four segmentation algorithms with the images having varied background. It is observed that the K-means method of segmentation gives the better values as JSC $=86 \%$ and DSC $=84 \%$. 
The Active contour Snake method gives the second best results i.e. $\mathrm{JSC}=84 \%$ and DSC $=81 \%$. The threshold method showed the values as JSC $=81 \%$ and DSC $=79 \%$. While the edge and convex hull combined method gives comparatively low values such as JSC $=69 \%$ and DSC $=62 \%$. Hence, the segmentation results are better for images having uniform background than cluttered background. Based on study of above explained algorithms the analytical comparison of segmentation techniques is elaborated in Table-III.

Table- I: The empirical comparison of algorithms

\begin{tabular}{|l|c|c|}
\hline & JSC & DSC \\
\hline Edge and Convex Hull & 0.92 & 0.91 \\
\hline Thresholding & 0.93 & 0.94 \\
\hline Active Contour (Snakes) & 0.94 & 0.93 \\
\hline K-means & $\mathbf{0 . 9 5}$ & $\mathbf{0 . 9 5}$ \\
\hline
\end{tabular}

Table- II: The empirical comparison of algorithms for simple varying background images.

\begin{tabular}{|l|c|c|}
\hline & JSC & DSC \\
\hline Edge and Convex Hull & 0.69 & 0.62 \\
\hline Thresholding & 0.81 & 0.79 \\
\hline Active Contour (Snakes) & 0.84 & 0.81 \\
\hline K-means & $\mathbf{0 . 8 6}$ & $\mathbf{0 . 8 4}$ \\
\hline
\end{tabular}

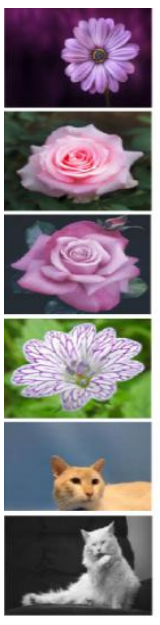

(a)
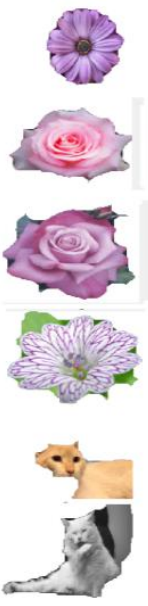

(b)

(c)
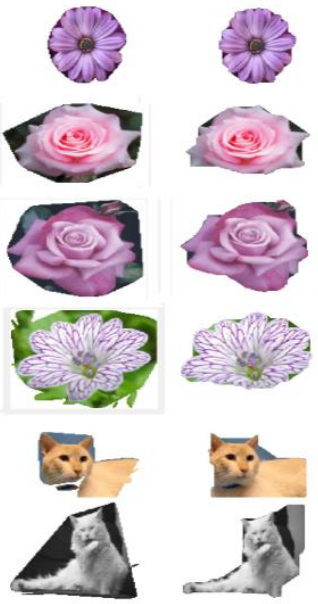

(d)

(e)
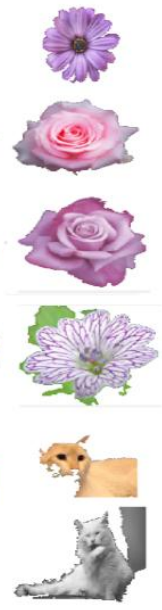

Fig 2: Results of four algorithms for simple varying background images(a) Input image (b) Threshold segmentation (c) Edge segmentation (d) K-means segmentation (e) Active Contour's segmentation.

\section{CONCLUSION}

This paper discussed the object segmentation algorithms for object based image retrieval. The segmentation is dependent on the type of object present and the background. Here, we have tried to segment objects from images with the help of different algorithms which are post processed in order to identify them better. The results obtained are discussed and tabulated. It was observed that with uniform background and cluttered background, K-means had performed better. In future, some more advanced algorithms with modifications have to be explored for the purpose of object based image retrieval.

Table- III: Analytical comparative study of proposed algorithms.

\begin{tabular}{|c|c|c|c|c|}
\hline & Global Thresholding & $\begin{array}{l}\text { Edge based object } \\
\text { segmentation with } \\
\text { convex hull. }\end{array}$ & $\begin{array}{l}\text { Active Contour } \\
\text { Snake's Model }\end{array}$ & K-means \\
\hline Method & Threshold & Edge & Object contour & Pixel clusters \\
\hline Approach & $\begin{array}{l}\text { Extracts the region of } \\
\text { interest from the } \\
\text { unwanted background by } \\
\text { using threshold } \mathrm{T} \text {. }\end{array}$ & $\begin{array}{l}\text { Partitions an image } \\
\text { through detecting } \\
\text { edge among } \\
\text { different regions. }\end{array}$ & $\begin{array}{l}\text { Detects the contour } \\
\text { based on an energy } \\
\text { minimization function } \\
\text { of Contour pixels. }\end{array}$ & $\begin{array}{ll}\text { Groups the pixels of } \\
\text { image into clusters } \\
\text { based on intensity } \\
\text { value. }\end{array}$ \\
\hline Pre-requisite & $\begin{array}{l}\text { Automatically detect } \\
\text { threshold and using that } \\
\text { binary image is found. }\end{array}$ & $\begin{array}{l}\text { Not necessary to } \\
\text { initialize any } \\
\text { parameters }\end{array}$ & $\begin{array}{l}\text { Need Initial rough } \\
\text { boundary pixel value to } \\
\text { start. }\end{array}$ & $\begin{array}{l}\text { Need Initial seed pixel } \\
\text { value as cluster centers } \\
\text { to start. }\end{array}$ \\
\hline
\end{tabular}




\begin{tabular}{|l|l|l|l|l|}
\hline Suitability & $\begin{array}{l}\text { If the object is well } \\
\text { distinct from the } \\
\text { background. }\end{array}$ & $\begin{array}{l}\text { If the object edges } \\
\text { are differentiable } \\
\text { from the } \\
\text { background. }\end{array}$ & $\begin{array}{l}\text { If the object edges are } \\
\text { differentiable from the } \\
\text { background. }\end{array}$ & $\begin{array}{l}\text { If the pixel values are } \\
\text { well separated. }\end{array}$ \\
\hline Advantage & Easy and basic method. & $\begin{array}{l}\text { Good and high-level } \\
\text { approach. }\end{array}$ & $\begin{array}{l}\text { Automatic search of } \\
\text { minimal energy curve. }\end{array}$ & $\begin{array}{l}\text { Easy to Visualize and } \\
\text { implement. }\end{array}$ \\
\hline Disadvantage & $\begin{array}{l}\text { A spatial relation among } \\
\text { the pixels values is is } \\
\text { ignored. Selecting } \\
\text { threshold value is not } \\
\text { always straightforward. }\end{array}$ & $\begin{array}{l}\text { Not suitable on the } \\
\text { smooth transitions } \\
\text { of image. }\end{array}$ & $\begin{array}{l}\text { Can get struck in local } \\
\text { minima states. }\end{array}$ & $\begin{array}{l}\text { The clustering depends } \\
\text { on the uninformed } \\
\text { selection of initial } \\
\text { centers. }\end{array}$ \\
\hline
\end{tabular}

\section{REFERENCES}

1. Yuheng Song, Hao Yan, "Image Segmentation Techniques Overview," Proc. Of Asia Modelling Symposium (AMS), pp. 103-107, 2017.

2. Jain, Sneha, and Vijaya Laxmi, "Color Image Segmentation Techniques: A Survey," Proceedings of the International Conference on Microelectronics, Computing \& Communication Systems, Springer, Singapore, 2018.

3. Zheng, Xin, Qinyi Lei, et al, "Image segmentation based on adaptive K-means algorithm," EURASIP Journal on Image and Video Processing, 2018.

4. Katare Aradhana, Suman K Mitra, and Asim Banerjee, "Content based image retrieval system for multi object images using combined features," International Conference on Computing: Theory and Applications (ICCTA'07). IEEE, 2007.

5. Sungyoung Kim, Soyoun Park, and Minhwan Kim, "Central object extraction for object-based image retrieval," International Conference on Image and Video Retrieval, Springer, Berlin, Heidelberg, 2003.

6. Ko, ByoungChul, and Hyeran Byun, "FRIP: a region-based image retrieval tool using automatic image segmentation and stepwise Boolean AND matching," IEEE Transactions on multimedia, 2005.

7. ChestiAltaff Hussain, D. Venkata Rao, and S. ArunaMasthani, "Robust pre-processing technique based on saliency detection for content based image retrieval systems," Procedia Computer Science 85, pp. 571-580, 2016.

8. Huang, Yin-Fu, and Bo-Rong Chen, "Content-Based Image Retrieval System for Real Images," 2nd Global Conference on Artificial Intelligence, 2016.

9. Zhang, Jun, And Jinglu Hu, "Image Segmentation Based On 2d Otsu Method With Histogram Analysis," 2008 International Conference on Computer Science and Software Engineering. Vol. 6. IEEE, 2008.

10. Joshi Shashidhar Ram, and Roshan Koju, "Study and comparison of edge detection algorithms," Third Asian Himalayas International Conference on Internet. IEEE, 2012.

11. Amer GMH, Abushaala AM, "Edge detection methods," Paper presented at: 2015 2ndWorld Symposium on Web Applications and Networking (WSWAN), 2015.

12. R. Chadnov, A. Skvortsov, "Convex hull algorithms review," Science and Technology KORUS 2004. Proceedings, The 8th Russian-Korean International Symposium on, vol. 2, pp. 112-115, 2004.

13. D. Baswaraj, A. Govardhan, and P. Premchand, "Active contours and image segmentation: The current state of the art," Global Journal of Computer Science and Technology, 2012.

14. Samaddar, Swapan, and A. R. Reddy, "Comparative study of image segmentation techniques on chronic kidney diseases," Int. J. Pure Appl. Math, 2018.

15. Savkare, S. S., A. S. Narote, and S. P. Narote, "Comparative Analysis of Segmentation Algorithms Using Threshold and K-Mean Clustering," The International Symposium on Intelligent Systems Technologies and Applications, Springer, Cham, 2016.
16. Shrivastava, Neeraj, and Jyoti Bharti, "Empirical analysis of image segmentation techniques," International Conference on Smart Trends for Information Technology and Computer Communications. Springer, Singapore, 2016

17. Polak Mark, Hong Zhang, and Minghong Pi, "An evaluation metric for image segmentation of multiple objects," Image and Vision Computing, 2009

\section{AUTHORS PROFILE}

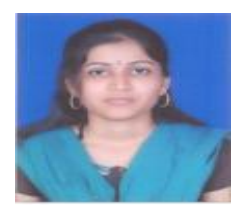

Mrs. LaxmideviNoolvi, she is currently pursuing her $\mathrm{Ph} . \mathrm{D}$ degree in the department of Computer Science and Engineering, RNSIT, Bangalore.

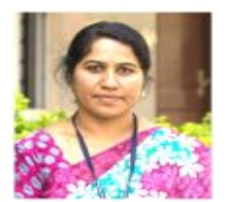

Mrs. Hema N, currently working as assistant Professor and pursuing Ph.D in Dept. of ISE, RNSIT. She is having Teaching experience of 10 years. She has knowledge in Medical Image Processing.

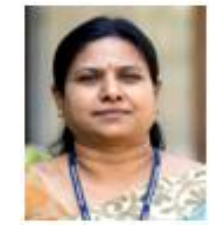

Dr. M V Sudhamani, currently working as Dean-R\&D, Professor and HoD, Dept. of ISE, RNSIT. She is having Teaching, Research and Industrial experience of 25 years. She has specialization in Image Processing, Content-based Image Retrieval, Advanced Algorithms and Databases. Guided and guiding candidates for $\mathrm{Ph}$. D degree. She has carried out two research projects from VTU and AICTE. She has served as member of Board of Examiners (BOE) and Board of Study (BOS) member in VTU and other autonomous institutions across India. She has organized two international conferences ICDECS 2011 and 2015, and one more in December 2019. 